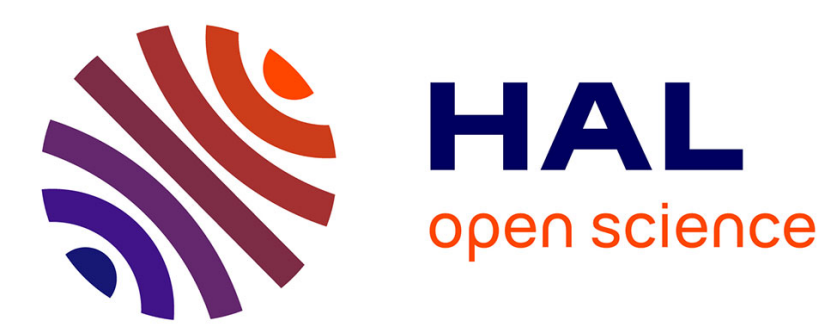

\title{
An augmented model for robust stability analysis of time-varying delay systems
}

Yassine Ariba, Frédéric Gouaisbaut

\section{To cite this version:}

Yassine Ariba, Frédéric Gouaisbaut. An augmented model for robust stability analysis of time-varying delay systems. International Journal of Control, 2009, 82 (9), p.1616-1626. hal-00357764

\section{HAL Id: hal-00357764 \\ https://hal.science/hal-00357764}

Submitted on 1 Feb 2009

HAL is a multi-disciplinary open access archive for the deposit and dissemination of scientific research documents, whether they are published or not. The documents may come from teaching and research institutions in France or abroad, or from public or private research centers.
L'archive ouverte pluridisciplinaire HAL, est destinée au dépôt et à la diffusion de documents scientifiques de niveau recherche, publiés ou non, émanant des établissements d'enseignement et de recherche français ou étrangers, des laboratoires publics ou privés. 


\title{
An augmented model for robust stability analysis of time-varying delay systems
}

\author{
Yassine Ariba*† and Frédéric Gouaisbaut**
}

October 2008

\begin{abstract}
Stability analysis of linear systems with time-varying delay is investigated. In order to highlight the relations between the variation of the delay and the states, redundant equations are introduced to construct a new modeling of the delay system. New types of Lyapunov Krasovskii functionals are then proposed allowing to reduce the conservatism of the stability criterion. Delay dependent stability conditions are then formulated in terms of linear matrix inequalities (LMI). Finally, several examples show the effectiveness of the proposed methodology.
\end{abstract}

\section{Introduction}

During the last decades, stability of linear time delay systems with constant delays have attracted a lot of attention, see for example [5], [12], [13], [16], and numerous tools for estimating the stability of such systems have been successfully exploited (see for example the book of [8]). Concerning the time varying case, results are much more scarce. Indeed, the methodology proposed by [17], [14] (and references therein) which exploits the characteristic equation cannot be applied. Only the Input-Output framework such as [6], [11], and [8] and the Lyapunov-krasovskii functional, see for example [5], [10] can be used. In the first issue, methods aim at embed the delay as an uncertain operator and hence transform the original delay system into a linear system submitted to a perturbation. Then, the use of classical robustness tools like Small Gain theorem, IQC allow then to develop effective criteria as in [6], [11]. In this framework, the source of induced conservatism is clear and generally comes from the choice of the interconnection (often related to the choice of a model transformation) and the choice of the uncertainty set which covers the delay operator. Here, the difference with the constant case is that the uncertainty set is also characterized by the upperbound of the delay derivative. Slower the time variation of the delay is, the less conservative the result are.

Another very popular approach relies on the use of a Lyapunov-Krasovskii functional. Indeed, even if for a linear time delay system with constant delay, a general functional can be found in [8], the time varying case is rather very

\footnotetext{
*Université de Toulouse; UPS, 118 Route de Narbonne, F-31062 Toulouse, France.

${ }^{\dagger}$ LAAS; CNRS; 7, avenue du Colonel Roche, F-31077 Toulouse, France. yariba@laas.fr
} 
difficult to handle. That is the reason why more simple and thus more conservative Lyapunov-Krasovskii functional have been proposed. Generally, all these approaches have to tackle with two main difficulties. The first one relies on the choice of the model transformation which is clearly related to the choice of the LKF. In the time-varying delay case, the LKF usually depends not only on the delay but also on an upperbound of the delay. The second problem lies on the bound of some cross terms which appears in the derivative of the Lyapunov functional. As it has been shown in [9],[10] and [1], ways to bound these latter terms have a deep impact on the conservatism of the proposed techniques. The present paper brings a contribution to the first issue using an augmented model of the time-varying delay systems, a method originally proposed by [4] for linear uncertain systems. For time delay systems, this fruitful idea has been adapted either for independent of delay case in [2] or delay dependent case, see [7]. It was shown that introducing redundant differential equations shifted in time allows to build conditions that improve results. For time varying delay, this technique has been partially used in [10], where an augmented Lyapunov functional is proposed which take into account the state variable and its derivative. The proposed theorem shows interesting results especially for robustness issues. In this paper, using the derivative operator, a different method is proposed to consider augmented time-varying delay systems and then to provide new delay dependent stability criteria. Based on this new modeling, new types of functionnals are proposed, which are proved to be less conservative that the Lyapunov functionals constructed formerly in the litterature.

The paper is organized as follows. In section 2, a first result is derived from a Lyapunov-Krasovskii functional developed in [9] for delay dependent stability analysis. This section aims at exhibiting another formulation of the analysis problem for time-varying delay systems. Then, in section 3 we expose the two main results of this paper: the use of the system derivative and an additional term for the Lyapunov-Krasovskii functional. Finally, the following section 4 is devoted to numerical examples that illustrate the proposed approach.

Notations: For two symmetric matrices, $A$ and $B, A>(\geq) B$ means that $A-B$ is (semi-) positive definite. $A^{T}$ denotes the transpose of $A$. $1_{\mathrm{n}}$ and $0_{\mathrm{m} \times \mathrm{n}}$ denote respectively the identity matrix of size $n$ and null matrix of size $m \times n$. If the context allows it, the dimensions of these matrices are often omitted. For a given matrix $B \in \mathrm{R}^{\mathrm{m} \times \mathrm{n}}$ such that $\operatorname{rank}(B)=r$, we define $B^{\perp} \in \mathrm{R}^{\mathrm{n} \times(\mathrm{n}-\mathrm{r})}$ the right orthogonal complement of $B$ by $B B^{\perp}=0$. We denote $x_{t}($.$) the state of$ a time-varying delay system by $x_{t}():. \theta \rightarrow x(t+\theta) \forall \theta \in\left[-h_{m}, 0\right]$ where $h_{m}$ is the maximal value of the delay. The formulation $X=\operatorname{diag}\left(X_{1}, X_{2}\right)$ denotes the block diagonal matrix $X=\left[\begin{array}{cc}X_{1} & 0 \\ 0 & X_{2}\end{array}\right]$.

\section{A first result on stability}

Consider the following linear time delay system:

$$
\begin{cases}\dot{x}(t)=A x(t)+A_{d} x(t-h(t)), & \forall t \geq 0, \\ x_{t}(\theta)=\phi(\theta), & \forall \theta \in\left[-h_{m}, 0\right],\end{cases}
$$

where $x(t) \in \mathrm{R}^{\mathrm{n}}$ is the instantaneous state vector, $A, A_{d} \in \mathrm{R}^{\mathrm{n} \times \mathrm{n}}$ are known constant matrices and $\phi$ is the initial condition. The delay, $h(t)$, is assumed to 
be a time-varying continuous function that satisfies

$$
0 \leq h(t) \leq h_{m},
$$

where $h_{m}>0$ may be arbitrarily large if delay independent conditions are looked for. Furthermore, we also assume that a bound on the derivative of $\dot{h}(t)$ is provided :

$$
|\dot{h}(t)| \leq d,
$$

The aim of this section is to derive some conditions on $h_{m}$, the upperbound which ensures the stability of (1) for a given value $d$ by using a LyapunovKrasovskii framework. The next theorem gives the following delay dependent result for system (1).

Theorem 1 Given scalars $h_{m}>0$ and $d \geq 0$, system (1) is asymptotically stable for any time-varying delay $h(t)$ satisfying (2) and (3) if there exists positive definite matrices $P, Q_{i}$ where $i=\{1,2\}$ and $R \in \mathrm{R}^{\mathrm{n} \times \mathrm{n}}$ such that the following LMI holds:

$$
S^{\perp^{T}} \Gamma S^{\perp}<0,
$$

where

$$
\Gamma=\left[\begin{array}{cccc}
h_{m} \mathbf{R} & \mathbf{P} & 0 & 0 \\
\mathbf{P} & T & \frac{1}{h_{m}} \mathbf{R} & 0 \\
0 & \frac{1}{h_{m}} \mathbf{R} & U & \frac{1}{h_{m}} \mathbf{R} \\
0 & 0 & \frac{1}{h_{m}} \mathbf{R} & V
\end{array}\right] \text { with } \begin{aligned}
T & =\mathbf{Q}_{\mathbf{1}}+\mathbf{Q}_{\mathbf{2}}-\frac{1}{h_{m}} \mathbf{R}, \\
& =-(1-d) \mathbf{Q}_{\mathbf{1}}-\frac{2}{h_{m}} \mathbf{R}, \\
V & =-\frac{1}{h_{m}} \mathbf{R}-\mathbf{Q}_{\mathbf{2}},
\end{aligned}
$$

and $S^{\perp}$ is a right orthogonal complement of $S$.

Proof 1 Define the following Lyapunov-Krasovskii functional candidate:

$$
\begin{aligned}
V\left(x_{t}\right) & =x_{t}^{T}(0) P x_{t}(0)+\int_{-h(t)}^{0} x_{t}^{T}(\theta) Q_{1} x_{t}(\theta) d \theta \\
& +\int_{-h_{m}}^{0} x_{t}^{T}(\theta) Q_{2} x_{t}(\theta) d \theta+\int_{t-h_{m}}^{0} \int_{\theta}^{0} \dot{x}_{t}^{T}(s) R \dot{x}_{t}(s) d s d \theta
\end{aligned}
$$

Remark that since $P, Q_{1}, Q_{2}, R$ are positive definite, we can conclude that for some $\epsilon>0$, the Lyapunov-Krasovskii functional condition $V\left(x_{t}\right) \geq \epsilon\left\|x_{t}(0)\right\|$ is satisfied, see for example [8]. The derivative along the trajectories of (1) leads to

$$
\begin{aligned}
\dot{V}\left(x_{t}\right)= & 2 x^{T}(t) P \dot{x}(t)+x^{T}(t) Q_{1} x(t)-(1-\dot{h}(t)) x^{T}(t-h(t)) Q_{1} x(t-h(t)) \\
& +x^{T}(t) Q_{2} x(t)-x^{T}\left(t-h_{m}\right) Q_{2} x\left(t-h_{m}\right)+h_{m} \dot{x}^{T}(t) R \dot{x}(t) \\
& -\int_{t-h_{m}}^{t} \dot{x}^{T}(\theta) R \dot{x}(\theta) d \theta .
\end{aligned}
$$


As noted in [9], the derivative of $\int_{t-h_{m}}^{t} \int_{\theta}^{t} \dot{x}^{T}(s) R \dot{x}(s) d s d \theta$ is often estimated as $h_{m} \dot{x}^{T}(t) R \dot{x}(t)-\int_{t-h(t)}^{t} \dot{x}^{T}(\theta) R \dot{x}(\theta) d \theta$ and the term $-\int_{t-h_{m}}^{t-h(t)} \dot{x}^{T}(\theta) R \dot{x}(\theta) d \theta$ is ignored, which may lead to considerable conservatism. Hence, the last term of (8) can be separated in two parts:

$$
-\int_{t-h_{m}}^{t} \dot{x}^{T}(\theta) R \dot{x}(\theta) d \theta=-\int_{t-h_{m}}^{t-h(t)} \dot{x}^{T}(\theta) R \dot{x}(\theta) d \theta-\int_{t-h(t)}^{t} \dot{x}^{T}(\theta) R \dot{x}(\theta) d \theta .
$$

Using the Jensen's inequality (see [8]), (9) can be bounded as follow:

$$
\begin{aligned}
-\int_{t-h_{m}}^{t-h(t)} \dot{x}^{T}(\theta) R \dot{x}(\theta) d \theta-\int_{t-h(t)}^{t} \dot{x}^{T}(\theta) R \dot{x}(\theta) d \theta & <v^{T}(t) \frac{R}{h_{m}-h(t)} v(t)-w^{T}(t) \frac{R}{h(t)} w(t) \\
& <-v^{T}(t) \frac{R}{h_{m}} v(t)-w^{T}(t) \frac{R}{h_{m}} w(t)
\end{aligned}
$$

with

$$
v(t)=x(t-h(t))-x\left(t-h_{m}\right) \text { and } w(t)=x(t)-x(t-h(t)) .
$$

Therefore, we get $\dot{V}\left(x_{t}\right)<\xi^{T}(t) \Gamma \xi(t)$ with $\Gamma$ defined as (6) and

$$
\xi(t)=\left[\begin{array}{c}
\dot{x}(t) \\
x(t) \\
x(t-h(t)) \\
x\left(t-h_{m}\right)
\end{array}\right] .
$$

Furthermore, using the extended variable $\xi(t)$, system (1) can be rewritten as $S \xi=0$ with $S$ defined as (5). The original system (1) is asymptotically stable if for all $\xi$ such that $S \xi=0$, the inequality $\xi^{T} \Gamma \xi<0$ holds. Using Finsler lemma, see [18], this is equivalent to $S^{\perp^{T}} \Gamma S^{\perp}<0$, where $S^{\perp}$ is a right orthogonal complement of $S$, this concludes the proof.

Note that Condition (4) can be rewritten as

$$
\begin{aligned}
& {\left[\begin{array}{ccc}
A^{T} P+P A+Q_{1}+Q_{2} & P A_{d} & 0 \\
A_{d}^{T} P & -(1-d) Q_{1} & 0 \\
0 & 0 & -Q_{2}
\end{array}\right]} \\
& -\frac{1}{h_{m}}\left[\begin{array}{cc}
-1 & 0 \\
1 & -1 \\
0 & 1
\end{array}\right]\left[\begin{array}{cc}
R & 0 \\
0 & R
\end{array}\right]\left[\begin{array}{cc}
-1 & 0 \\
1 & -1 \\
0 & 1
\end{array}\right]^{T}+h_{m}\left[\begin{array}{c}
A^{T} \\
A_{d}^{T} \\
0
\end{array}\right] R\left[\begin{array}{c}
A^{T} \\
A_{d}^{T} \\
0
\end{array}\right]^{T}<0
\end{aligned}
$$

if $S^{\perp}$ is expressed as

$$
S^{\perp}=\left[\begin{array}{ccc}
A & A_{d} & 0 \\
1 & 0 & 0 \\
0 & 1 & 0 \\
0 & 0 & 1
\end{array}\right]
$$

Thus, according to this latter expression, we can conclude that if the LMI (11) is feasible for a given $h_{m}>0$, then it is feasible also for all delays less than the prescribed upperbound $h_{m}$.

Remark 1 Instead of using an orthogonal complement of S, Finsler lemma also states that condition $S^{\perp^{T}} \Gamma S^{\perp}<0$ is equivalent to the existence of some $X \in$ 
$\mathrm{R}^{4 \mathrm{n} \times \mathrm{n}}$ such that the $L M I \Gamma+X S+S^{T} X^{T}<0$ holds. Creating such additional variable $X$ is useless for the considered case: it only increases the number of variables and constraints in the LMI problem without reducing conservatism of the approach. But as demonstrated in [15], [7] and many others, such additional "slack variables" are of major interest for robust analysis purpose.

Remark 2 Note that delay-dependent results for fast varying delay (i.e. proving stability whatever the positive bound d) are a special case of the theorem 1. Fixing $Q_{1}=0$ provides the conditions independent on $d$ and therefore gives conditions for possibly fast varying delays.

\section{Main results}

\subsection{An augmented state for modelling the delayed systems}

Actually, Theorem 1 is not a new result but rather a new formulation of existing equivalent results with fewer decision variables. Here, we aim at developing further the methodology used in the previous section to derive less conservative results. The key idea is that since the proposed delay-dependent criterion depends also on the derivative of the delay, we should highlight the relation between $\dot{h}(t)$ and states variables. One way is to consider an extended state $z=\left[\begin{array}{ll}x^{T} & \dot{x}^{T}\end{array}\right]^{T}$.

Differentiating the system (1), we get:

$$
\ddot{x}(t)=A \dot{x}(t)+(1-\dot{h}(t)) A_{d} \dot{x}(t-h(t)) .
$$

Indeed, introducing derivative of the differential equation (1) provides directly a link between the state variables and $\dot{h}(t)$. Considering the artificially augmented system

$$
\left\{\begin{array}{l}
\dot{x}(t)=A x(t)+A_{d} x(t-h(t)) \\
\ddot{x}(t)=A \dot{x}(t)+(1-\dot{h}(t)) A_{d} \dot{x}(t-h(t))
\end{array},\right.
$$

introducing the augmented state

$$
z(t)=\left[\begin{array}{c}
x(t) \\
\dot{x}(t)
\end{array}\right]
$$

and specifying the relationship between the two components of $z(t)$ with the equality $\left[\begin{array}{ll}1 & 0\end{array}\right] \dot{z}(t)=\left[\begin{array}{ll}0 & 1\end{array}\right] z(t)$, we get the new augmented system

$$
E \dot{z}(t)=\bar{A} z(t)+\bar{A}_{d} z(t-h(t)),
$$

where

$$
E=\left[\begin{array}{ll}
1 & 0 \\
0 & 1 \\
1 & 0
\end{array}\right], \quad \bar{A}=\left[\begin{array}{cc}
A & 0 \\
0 & A \\
0 & 1
\end{array}\right], \quad \overline{A_{d}}=\left[\begin{array}{cc}
A_{d} & 0 \\
0 & (1-\dot{h}(t)) A_{d} \\
0 & 0
\end{array}\right]
$$

Finally, we obtain a descriptor linear time delay and time varying system, which may be more difficult to handle. A first idea would be to apply the methodology developped in Section 2 except that the stability would be 
guaranteed only for a fixed $\dot{h}(t)$ since this term appears in $\bar{A}_{d}$. A possible solution consists then in embedding the time varying parameters $h$ and $\dot{h}$ into an uncertain set, described by a polytopic set and employing quadratic stability framework (see [3]). This approach is proposed in Theorem 2:

Theorem 2 Let define matrices $\mathcal{A}, \mathcal{B}$ and $\Theta_{2}$ as

$$
\begin{aligned}
& \mathcal{A}(\dot{h})=\Theta_{1}^{T} P \Theta_{2}(\dot{h})+\Theta_{2}^{T}(\dot{h}) P \Theta_{1}+\Theta_{3}^{T}\left[\begin{array}{cc}
Q_{1} & 0 \\
0 & -(1-\dot{h}) Q_{1}
\end{array}\right] \Theta_{3}+\Theta_{4}^{T}\left[\begin{array}{cc}
Q_{2} & 0 \\
0 & -Q_{2}
\end{array}\right] \Theta_{4}, \\
& \mathcal{B}=\Theta_{5}^{T}\left[\begin{array}{cc}
R & 0 \\
0 & R
\end{array}\right] \Theta_{5} \text { and } \Theta_{2}(\dot{h})=\left[\begin{array}{ccccc}
A & A_{d} & 0 & 0 & 0 \\
A^{2} & A A_{d} & (1-\dot{h}) A_{d} & 0 & 0
\end{array}\right] \text {, }
\end{aligned}
$$

where

$$
\begin{aligned}
& \Theta_{1}=\left[\begin{array}{ccccc}
1 & 0 & 0 & 0 & 0 \\
A & A_{d} & 0 & 0 & 0
\end{array}\right], \Theta_{3}=\left[\begin{array}{ccccc}
1 & 0 & 0 & 0 & 0 \\
A & A_{d} & 0 & 0 & 0 \\
0 & 1 & 0 & 0 & 0 \\
0 & 0 & 1 & 0 & 0
\end{array}\right], \\
& \Theta_{4}=\left[\begin{array}{ccccc}
1 & 0 & 0 & 0 & 0 \\
A & A_{d} & 0 & 0 & 0 \\
0 & 0 & 0 & 1 & 0 \\
0 & 0 & 0 & 0 & 1
\end{array}\right], \Theta_{5}=\left[\begin{array}{ccccc}
0 & 1 & 0 & -1 & 0 \\
0 & 0 & 1 & 0 & -1 \\
1 & -1 & 0 & 0 & 0 \\
A & A_{d} & -1 & 0 & 0
\end{array}\right] .
\end{aligned}
$$

Given scalars $h_{m}>0$ and $d \geq 0$, system (1) is asymptotically stable for any time-varying delay $h(t)$ satisfying (2) and (3) if there exists positive definite matrices $P, Q_{j}$ where $j=\{1,2\}$ and $R \in \mathrm{R}^{2 \mathrm{n} \times 2 \mathrm{n}}$ such that the following LMI holds for $i=\{1,2\}$ :

$$
\left[\begin{array}{cc}
\mathcal{A}^{(i)}-\frac{1}{h_{m}} \mathcal{B} & \Theta_{2}^{(i)^{T}} \mathbf{R} \\
\mathbf{R} \Theta_{2}^{(i)} & -\frac{1}{h_{m}} \mathbf{R}
\end{array}\right]<0
$$

where $\mathcal{A}^{(i)}$ and $\Theta_{2}^{(i)}$ for $i=1,2$ are the two vertices of $\mathcal{A}(\dot{h}) \in \mathrm{R}^{5 \mathrm{n} \times 5 \mathrm{n}}$ and $\Theta_{2}(\dot{h}) \in \mathrm{R}^{2 \mathrm{n} \times 5 \mathrm{n}}$ respectively, replacing the term $\dot{h}(t)$ by $d_{i} . d_{i}, i=\{1,2\}$ corresponding to the bounds of $\dot{h}(t): d_{1}=d$ and $d_{2}=-d$.

Proof 2 We now consider the following Lyapunov-Krasovskii functional associated with the augmented state vector $z(t)$ :

$$
V\left(z_{t}\right)=z_{t}^{T}(0) P z_{t}(0)+\int_{-h(t)}^{0} z_{t}^{T}(\theta) Q_{1} z_{t}(\theta) d \theta+\int_{-h_{m}}^{0} z_{t}^{T}(\theta) Q_{2} z_{t}(\theta) d \theta+\int_{-h_{m}}^{0} \int_{\theta}^{0} \dot{z}_{t}^{T}(s) R \dot{z}_{t}(s) d s d \theta .
$$

Using the same idea developed in the proof of Theorem 1, the derivative of (20) is such that $\dot{V}\left(z_{t}\right) \leq \psi(t)^{T} \Gamma(\dot{h}) \psi(t)$ where

$$
\psi(t)=\left[\begin{array}{c}
\dot{z}(t) \\
z(t) \\
z(t-h(t)) \\
z\left(t-h_{m}\right)
\end{array}\right], \Gamma(\dot{h})=\left[\begin{array}{cccc}
h_{m} R & P & 0 & 0 \\
P & T & \frac{1}{h_{m}} R & 0 \\
0 & \frac{1}{h_{m}} R & U & \frac{1}{h_{m}} R \\
0 & 0 & \frac{1}{h_{m}} R & V
\end{array}\right]
$$


with

$$
\begin{aligned}
T & =Q_{1}+Q_{2}-\frac{1}{h_{m}} R, \\
U & =-(1-\dot{h}(t)) Q_{1}-\frac{2}{h_{m}} R, \\
V & =-\frac{1}{h_{m}} R-Q_{2} .
\end{aligned}
$$

So, system (15) is asymptotically stable if for all $\psi$ such that $S(\dot{h}) \psi=0$ with

$$
S(\dot{h})=\left[\begin{array}{llll}
-E & \bar{A} & \bar{A}_{d} & 0
\end{array}\right],
$$

the inequality $\psi(t)^{T} \Gamma(\dot{h}) \psi(t)<0$ holds. Using Finsler lemma, this is equivalent to

$$
S^{\perp^{T}}(\dot{h}) \Gamma(\dot{h}) S^{\perp}(\dot{h})<0
$$

where $S^{\perp}(\dot{h})$ is a right orthogonal complement of $S(\dot{h})$ given by

$$
S^{\perp}(\dot{h})=\left[\begin{array}{ccccc}
A & A_{d} & 0 & 0 & 0 \\
A A & A A_{d} & (1-\dot{h}) A_{d} & 0 & 0 \\
1 & 0 & 0 & 0 & 0 \\
A & A_{d} & 0 & 0 & 0 \\
0 & 1 & 0 & 0 & 0 \\
0 & 0 & 1 & 0 & 0 \\
0 & 0 & 0 & 1 & 0 \\
0 & 0 & 0 & 0 & 1
\end{array}\right] .
$$

Carrying out algebraic calculus of (23) with (24), condition (25) is derived:

$$
\mathcal{A}(\dot{h})-\frac{1}{h_{m}} \mathcal{B}+h_{m} \Theta_{2}^{T}(\dot{h}) R \Theta_{2}(\dot{h})<0
$$

where matrices $\mathcal{A}, \mathcal{B}$ and $\Theta_{2}$ are defined as (17) and (18). Since matrix $R$ is positive definite and using schur complement, condition (25) is equivalent to

$$
\left[\begin{array}{cc}
\mathcal{A}(\dot{h})-\frac{1}{h_{m}} \mathcal{B} & \Theta_{2}^{T}(\dot{h}) \mathbf{R} \\
\mathbf{R} \Theta_{2}(\dot{h}) & -\frac{1}{h_{m}} \mathbf{R}
\end{array}\right]<0
$$

At this stage, assume that $\dot{h}(t)$ is not precisely known but varies between a lower and upper bound, $\dot{h}(t) \in[-d, d]$. Note that it exists $\alpha_{1}$ and $\alpha_{2}$ with $\alpha_{1}(t)+\alpha_{2}(t)=1$ such that $\dot{h}(t)$ can be written as $\dot{h}(t)=\alpha_{1}(t) d-\alpha_{2}(t) d$. Since this uncertain parameter appears linearly in (26), the uncertain set can be described by a polytope (see [3]). The vertices of this set can be calculated by setting the parameter to either lower or upper limit. Hence, condition (26) becomes:

$$
\left[\begin{array}{cc}
\sum_{i=1}^{2} \alpha_{i} \mathcal{A}^{(i)}-\frac{1}{h_{m}} \mathcal{B} & \sum_{i=1}^{2} \alpha_{i} \Theta_{2}^{(i)^{T}} \mathbf{R} \\
\mathbf{R} \sum_{i=1}^{2} \alpha_{i} \Theta_{2}^{(i)} & -\frac{1}{h_{m}} \mathbf{R}
\end{array}\right]<0
$$

where $\alpha_{i}(t) \in[0,1], \sum_{i=1}^{2} \alpha_{i}(t)=1, \mathcal{A}^{(i)}$ and $\Theta_{2}^{(i)}$ with $i=1,2$ are the two vertices of the uncertain matrices $\mathcal{A}(\dot{h})$ and $\Theta_{2}(\dot{h})$ respectively for $\dot{h}(t) \in[-d, d]$. Considering the quadratic stability framework as presented in [3], a sufficient condition to ensure (27) is

$$
\left[\begin{array}{cc}
\mathcal{A}^{(i)}-\frac{1}{h_{m}} \mathcal{B} & \Theta_{2}^{(i)^{T}} \mathbf{R} \\
\mathbf{R} \Theta_{2}^{(i)^{2}} & -\frac{1}{h_{m}} \mathbf{R}
\end{array}\right]<0, \mathrm{i}=1,2 .
$$


Thus, the inequality (26) has to be verified only on its vertices (28). Finally, the asymptotic stability of system (15) is guaranteed if the two LMI (28) are feasible with the same Lyapunov matrices. For any initial conditions, the whole state $z(t)$ converges asymptotically to zero. Its components $x(t)$ converge as well. The original system (1) is asymptotically stable.

Remark 3 In the same way that in Section 2 for Theorem 1, if condition (25) holds for $h_{m}$ then it still holds for $h(t) \leq h_{m}$.

It is worthy to note that considering the LKF (20) and assigning to $P, Q_{1}, Q_{2}$ and $R$ the particular choice $\left[\begin{array}{cc}P_{11} & 0 \\ 0 & 0\end{array}\right],\left[\begin{array}{cc}Q_{1_{11}} & 0 \\ 0 & 0\end{array}\right],\left[\begin{array}{cc}Q_{2_{11}} & 0 \\ 0 & 0\end{array}\right]$ and $\left[\begin{array}{cc}R_{11} & 0 \\ 0 & 0\end{array}\right]$, respectively, the recent results of [9], [10] are recovered. Moreover, if $Q_{2_{11}}$ is set to 0 and the separation of the integral is not performed as explained in [9] (i.e. considering both terms in $-\int_{t-h(t)}^{t} \dot{x}^{T}(u) R \dot{x}(u) d u-$ $\int_{t-h_{m}}^{t-h(t)} \dot{x}^{T}(u) R \dot{x}(u) d u$ ) (see (9)), the classical results of the literature [5] [19] [11] are obtained. Consequently, criteria provided in this paper are necessarily less pessimistic in the sense that results obtained are at least equivalent to the traditional stability conditions. We can propose the following:

Corollary 1 If the system (1) is proved to be stable with the results of the literature based on $\operatorname{LKF}(7)$, see for example [5], [19] then condition (19) of Theorem 2 is also satisfied.

\subsection{A new Lyapunov functional}

The proposed new functional is based on the extension of a classical LyapunovKrasovskii functional (7). In order to take into account the variable $\ddot{x}(t)$, let introduce a new term for the Lyapunov-Krasovskii functional.

$$
\begin{aligned}
& V\left(z_{t}\right)=z_{t}^{T}(0) P z_{t}(0)+\int_{-h(t)}^{0} z_{t}^{T}(\theta) Q z_{t}(\theta) d \theta \\
& +\int_{-h_{m}}^{0} \int_{\theta}^{0} \dot{z}_{t}^{T}(s) R \dot{z}_{t}(s) d s d \theta+\int_{-h_{m}}^{0} \iint_{s}^{0} \ddot{x}_{t}^{0} \ddot{x}_{t}^{T}(\theta) W \ddot{x}_{t}(\theta) d \theta d u d s
\end{aligned}
$$

Using this latter functional, the following result is proposed.

Theorem 3 Given scalars $h_{m}>h_{\min }>0, d \geq 0$, system (1) is asymptotically stable for any time-varying delay $h(t)$ satisfying (2) and (3) if there exists definite positive matrices $P, Q, R \in \mathrm{R}^{2 \mathrm{n} \times 2 \mathrm{n}}$, a definite positive matrix $W \in \mathrm{R}^{\mathrm{n} \times \mathrm{n}}$ and a matrix $X \in \mathrm{R}^{7 \mathrm{n} \times 4 \mathrm{n}}$ such that the following $L M I$ holds for $i=\{1, \ldots, 4\}$ :

$$
\begin{aligned}
& {\left[\begin{array}{ccc}
\Gamma & \mathcal{E}^{T} \Theta_{3}^{(i)^{T}} \mathbf{R} & \mathcal{E}^{T} \Theta_{3}^{(i)^{T}} E_{2}^{T} \mathbf{W} \\
\mathbf{R} \Theta_{3}^{(i)} \mathcal{E} & -\frac{1}{h_{m}} \mathbf{R} & 0 \\
\mathbf{W} E_{2} \Theta_{3}^{(i)} \mathcal{E} & 0 & -\frac{2}{h_{m}^{2}} \mathbf{W}
\end{array}\right]<0} \\
& \Gamma=\mathcal{A}^{(i)}+\mathbf{X} S^{(i)}+S^{(i)^{T}} \mathbf{X}^{T}
\end{aligned}
$$

where $\mathcal{A}^{(i)}, \Theta_{3}^{(i)}$ and $S^{(i)}$ for $i=\{1, \ldots, 4\}$ are the vertices of matrices $\mathcal{A}(h, \dot{h}) \in$ $\mathrm{R}^{7 \mathrm{n} \times 7 \mathrm{n}}, \Theta_{3}(\dot{h}) \in \mathrm{R}^{2 \mathrm{n} \times 2 \mathrm{n}}$ and $S(h, \dot{h}) \in \mathrm{R}^{4 \mathrm{n} \times 7 \mathrm{n}}$ respectively, replacing the terms $h$ 
by $h_{\min }$ and $h_{m}$ and $\dot{h}(t)$ by $d$ and $-d$. $\mathcal{A}, S, \mathcal{E}$ and $\Theta_{3}$ are defined as

$$
\begin{aligned}
S(h, \dot{h}) & =\left[\begin{array}{ccccccc}
1 & -1 & 0 & -h 1 & 0 & 0 & 0 \\
A & A_{d} & -1 & 0 & -h 1 & 0 & 0 \\
A & A_{d} & 0 & -1 & 0 & -1 & 0 \\
A^{2} & A A_{d} & (1-\dot{h}) A_{d} & 0 & -1 & 0 & -1
\end{array}\right], \Theta_{3}(\dot{h})=\left[\begin{array}{cc}
0 & 0 \\
(1-\dot{h}) A_{d} & 0
\end{array}\right], \\
\mathcal{A}(h, \dot{h}) & =N^{T} M N-\mathcal{E}^{T} \Theta_{3}^{T}(\dot{h}) T \Theta_{3}(\dot{h}) \mathcal{E}, \quad \mathcal{E}=\left[\begin{array}{cccc}
0_{2 n} & 1_{2 n} & 0_{2 n} & 0_{n} \times 2 \mathrm{n}
\end{array}\right] .
\end{aligned}
$$

Proof 3 First, let define the two matrices $E_{1}=\left[\begin{array}{ll}1_{n} & 0_{n}\end{array}\right]$ and $E_{2}=\left[\begin{array}{ll}0_{n} & 1_{n}\end{array}\right]$. Consider the Lyapunov-Krasovskii functional (29). The calculus of its derivative leads to:

$$
\begin{aligned}
\dot{V}= & 2 \dot{z}^{T}(t) P z(t)+z^{T}(t) Q z(t) \\
& -(1-\dot{h}(t)) z^{T}(t-h(t)) Q z(t-h(t))+h_{m} \dot{z}^{T}(t) R \dot{z}(t) \\
& -\int_{-h(t)}^{0} z_{t}(\theta)^{T} R z_{t}(\theta) d \theta+\frac{h_{m}^{2}}{2} \dot{z}^{T} E_{2}^{T} W E_{2} \dot{z}-\int_{-h(t)}^{0} \int_{s}^{0} x_{t}(\theta)^{T} W x_{t}(\theta) d \theta d s .
\end{aligned}
$$

Then, applying the Jensen's inequality, an upperbound of the derivative is derived:

$$
\begin{aligned}
\dot{V} \leq & 2 \dot{z}^{T}(t) P z(t)+z^{T}(t) Q z(t)-(1-\dot{h}(t)) z^{T}(t-h(t)) Q z(t-h(t)) \\
& +h_{m} \dot{z}^{T}(t) R \dot{z}(t)-\int_{-h(t)}^{0} z_{t}(\theta)^{T} d \theta \frac{R}{h(t)} \int_{-h(t)}^{0} z_{t}(\theta) d \theta \\
& +\frac{h_{m}^{2}}{2} \dot{z}^{T} E_{2}^{T} W E_{2} \dot{z}-\int_{-h(t)}^{0} \int_{s}^{0} x_{t}(\theta)^{T} d \theta \frac{W}{\int_{-h(t)}^{0} \int_{s}^{0} d \theta d s} \int_{-h(t)}^{0} \int_{s}^{0} x_{t}(\theta) d \theta d s,
\end{aligned}
$$

that is equivalent to

$$
\begin{aligned}
\dot{V} \leq & 2 \dot{z}^{T}(t) P z(t)+z^{T}(t) Q z(t) \\
& -(1-\dot{h}(t)) z^{T}(t-h(t)) Q z(t-h(t))+h_{m} \dot{z}^{T}(t) R \dot{z}(t) \\
& -[z(t)-z(t-h(t))]^{T} \frac{R}{h(t)}[z(t)-z(t-h(t))]+\frac{h_{m}^{2}}{2} \dot{z}^{T} E_{2}^{T} W E_{2} \dot{z} \\
& -\left[h(t) E_{1} \dot{z}(t)-E_{1}(z(t)-z(t-h(t)))\right]^{T} \frac{W}{h^{2}(t) / 2}\left[h(t) E_{1} \dot{z}(t)-E_{1}(z(t)-z(t-h(t)))\right] .
\end{aligned}
$$

Notice that the last term of the inequality is not linear with respect to $h(t)$. Nevertheless introducing the following signals

$$
\delta_{1}(t)=\frac{z(t)-z(t-h(t)))}{h(t)} \text { and } \delta_{2}(t)=\dot{z}(t)-\delta_{1},
$$

allows to transform the right hand side of (32) into

$$
\begin{aligned}
\dot{V} \leq & 2 \dot{z}^{T}(t) P z(t)+z^{T}(t) Q z(t)-(1-\dot{h}(t)) z^{T}(t-h(t)) Q z(t-h(t)) \\
& +h_{m} \dot{z}^{T}(t) R \dot{z}(t)-h(t) \delta_{1}^{T}(t) R \delta_{1}(t)+\frac{h_{m}^{2}}{2} \dot{z}^{T} E_{2}^{T} W E_{2} \dot{z}-2 \delta_{2}^{T}(t) E_{1}^{T} W E_{1} \delta_{2}(t) .
\end{aligned}
$$


Defining two extended vectors:

$$
\xi(t)=\left[\begin{array}{c}
z(t) \\
\dot{z}(t) \\
z(t-h(t)) \\
\delta_{1}(t) \\
\delta_{2}(t)
\end{array}\right] \text { and } \psi(t)=\left[\begin{array}{c}
x(t) \\
z(t-h(t)) \\
\delta_{1}(t) \\
\delta_{2}(t)
\end{array}\right]
$$

the equation (34) can be expressed as

$$
\dot{V} \leq \xi^{T} \underbrace{\left[\begin{array}{ccccc}
Q & P & 0 & 0 & 0 \\
P & T & 0 & 0 & 0 \\
0 & 0 & -(1-\dot{h}) Q & 0 & 0 \\
0 & 0 & 0 & -h R & 0 \\
0 & 0 & 0 & 0 & -2 E_{1}^{T} W E_{1}
\end{array}\right]}_{M} \xi
$$

with $T=h_{m} R+\frac{h_{m}^{2}}{2} E_{2}^{T} W E_{2}$. Then, using expressions of $\dot{x}$ and $\ddot{x}$ the following inequality is deduced.

$$
\dot{V} \leq \psi^{T}(t) N^{T} M N \psi(t)
$$

where $M$ is the matrix of the inequality (36) and

$N=\left[\begin{array}{cccc}\Theta_{1} & 0_{2 n} & 0_{2 n} & 0_{2 n \times n} \\ \Theta_{2} & \Theta_{3}(\dot{h}) & 0_{2 n} & 0_{2 n \times n} \\ 0_{2 n \times n} & 1_{2 n} & 0_{2} & 0_{2 n} \\ 0_{2 n \times n} & 0_{2 n} & 1_{2} & 0_{2 n} \\ 0_{2 n \times n} & 0_{2 n} & 0_{2} & 1_{2 n}\end{array}\right]$ with $\Theta_{1}=\left[\begin{array}{cc}1 & 0 \\ A & A_{d}\end{array}\right], \quad \Theta_{2}=\left[\begin{array}{cc}A & A_{d} \\ A^{2} & A A_{d}\end{array}\right]$,

and $\Theta_{3}$ defined as (31). So, the stability of the system can be ensured if the inequality $N^{T} M N<0$ under the constraint $S \psi=0$ with $S$ expressed as (31) holds. Thus, using Finsler's lemma, a sufficient condition to prove the stability is to verify that there exists $X \in \mathrm{R}^{7 \mathrm{n} \times 4 \mathrm{n}}$ such that:

$$
\mathcal{A}(h, \dot{h})+X S(h, \dot{h})+S^{T}(h, \dot{h}) X^{T}+\mathcal{E}^{T} \Theta_{3}^{T}(\dot{h}) T \Theta_{3}(\dot{h}) \mathcal{E} \leq 0
$$

with $\mathcal{A}$ and $\mathcal{E}$ defined as (31) is satisfied.

Then, applying twice the Schur's complement, expression (30) of Theorem 3 is obtained. Since $h$ and $\dot{h}$ appear linearly in (30) and using similar arguments as in the proof of Theorem 2, if the condition (30) is satisfied then the system (15) is asymptotically stable. As previously, since the whole state $z$ converges asymptotically to zero, its first component $x$ converges as well.

Remark 4 Note that, conditions (19) and (30) from Theorem 2 and Theorem 3, respectively, can be presented in equivalent different expressions. Indeed, applying Finsler's lemma directly to (23) and (36), respectively, additional slack variables are introduced. These criteria are easier to derive but multipliers involve much more decision variables and thus increases the processing time cost. However, it is an interesting alternative for robustness issues (see Section 4) and design problems. 


\section{Robustness issues}

The proposed approach in Section 3 is now extended to the robust case by considering affine polytopic uncertain models:

$$
\dot{x}(t)=A(\alpha) x(t)+A_{d}(\alpha) x(t-h(t)),
$$

with $h(t)$ satisfying conditions (2) and (3), [ $\left.\begin{array}{ll}A(\alpha) & A_{d}(\alpha)\end{array}\right]=\sum_{i=1}^{\eta} \alpha_{i}\left[\begin{array}{ll}A^{[i]} & A_{d}^{[i]}\end{array}\right]$ where $\alpha=\left(\alpha_{1}, \ldots, \alpha_{\eta}\right)$ belongs to the set

$$
\Xi=\left\{\alpha_{i} \geq 0, \quad \sum_{i=1}^{\eta} \alpha_{i}=1\right\}
$$

The uncertain model is assumed to be invariant w.r.t. time. Based on this latter assumption, the derivative of the differential equation (40) is of the form

$$
\ddot{x}(t)=A(\alpha) \dot{x}(t)+(1-\dot{h}(t)) A_{d}(\alpha) \dot{x}(t-h(t)) .
$$

Based on equations (40), (42) and the extended state $z(t)$ defined in (14), a new modeling of uncertain time-varying delay systems is constructed as follows

$$
E \dot{z}(t)=\bar{A}(\alpha) z(t)+\bar{A}_{d 1}(\alpha) z(t-h(t))+\bar{A}_{d 2}(\alpha) w(t),
$$

where

$E=\left[\begin{array}{ll}1 & 0 \\ 0 & 1 \\ 1 & 0\end{array}\right], \quad \bar{A}_{d 1}(\alpha)=\left[\begin{array}{cc}A_{d}(\alpha) & 0 \\ 0 & A_{d}(\alpha) \\ 0 & 0\end{array}\right], \bar{A}(\alpha)=\left[\begin{array}{cc}A(\alpha) & 0 \\ 0 & A(\alpha) \\ 0 & 1\end{array}\right], \quad \bar{A}_{d 2}(\alpha)=\left[\begin{array}{cc}0 & 0 \\ 0 & A_{d}(\alpha) \\ 0 & 0\end{array}\right]$

with $w(t)=-\dot{h}(t) z(t-h(t))$. This artificially augmented system and the change of variable allow us to apply our previously exposed methodology and we propose the following theorem.

Theorem 4 Given scalars $h_{m}>0, d \geq 0$, the linear uncertain system (40) is asymptotically robustly stable for any time-varying delay $h(t)$ satisfying (2), (3) and $\alpha \in \Xi$ (41) if there exists definite positive matrices $P^{[i]}, Q^{[i]}, Q_{m}^{[i]}$, $R^{[i]} \in \mathrm{R}^{2 \mathrm{n} \times 2 \mathrm{n}}$, a definite positive matrix $W^{[i]} \in \mathrm{R}^{\mathrm{n} \times \mathrm{n}}$ and a matrix $X \in \mathrm{R}^{14 \mathrm{n} \times 9 \mathrm{n}}$ such that the following LMI holds for $i=\{1, \ldots, \eta\}, j=\{1,2\}$ and $k=\{1,2\}$ :

$$
\Gamma^{[i]}+\mathbf{X} S^{[i][j][k]}+S^{[i][j][k]^{T}} \mathbf{X}^{T}<0
$$

where

$$
\begin{aligned}
& \boldsymbol{\Gamma}^{[i]}=\operatorname{diag}\left(\left[\begin{array}{ll}
\mathbf{Q}^{[i]} & \mathbf{P}^{[i]} \\
\mathbf{P}^{[i]} & \mathbf{T}^{[i]}
\end{array}\right],\left[\begin{array}{cc}
-(1-d) \mathbf{Q}^{[i]}-\frac{1}{h_{m}} R^{[i]} & \frac{1}{h_{m}} R^{[i]} \\
\frac{1}{h_{m}} R^{[i]} & -Q_{m}^{[i]}-\frac{1}{h_{m}} R^{[i]}
\end{array}\right], 0_{2 \mathrm{n}},-\mathbf{h}_{\mathbf{m}} \mathbf{R}^{[i]},-2 \mathbf{E}_{1}^{\top} \mathbf{W}^{[i]} \mathbf{E}_{1}\right), \\
& \mathbf{T}^{[i]}=h_{m} \mathbf{R}^{[i]}+\frac{h_{m}^{2}}{2} E_{2}^{T} \mathbf{W}^{[i]} E_{2}, \\
& S^{[i][j][k]}=\left[\begin{array}{ccccccc}
\bar{A}^{[i]} & -E & \bar{A}_{d 1}^{[i]} & 0 & \bar{A}_{d 2}^{[i]} & 0 & 0 \\
0 & 0 & \dot{h}^{[k]} 1 & 0 & 1 & 0 & 0 \\
1 & 0 & -1 & 0 & 0 & -h^{[j]} 1 & 0 \\
0 & 1 & 0 & 0 & 0 & -1 & -1
\end{array}\right], \quad \bar{A}^{[i]}=\left[\begin{array}{cc}
A^{[i]} & 0 \\
0 & A^{[i]} \\
0 & 1
\end{array}\right] \text {, } \\
& \bar{A}_{d 1}^{[i]}=\left[\begin{array}{cc}
A_{d}^{[i]} & 0 \\
0 & A_{d}^{[i]} \\
0 & 0
\end{array}\right], \quad \bar{A}_{d 2}^{[i]}=\left[\begin{array}{cc}
0 & 0 \\
0 & A_{d}^{[i]} \\
0 & 0
\end{array}\right], \quad\left\{\begin{array}{l}
h^{[1]}=0 \text { and } h^{[2]}=h_{m} \\
\dot{h}^{[1]}=-d \text { and } \dot{h}^{[2]}=d
\end{array}\right.
\end{aligned}
$$


with $A^{[i]}$ and $A_{d}^{[i]}$ for $i=\{1, \ldots, \eta\}$ are the vertices of the matrices $A(\alpha)$ and $A_{d}(\alpha)$ according to the polytopic uncertainty.

Proof 4 In order to derived an efficient criterion the principle of parameters dependent Lyapunov function is considered. Let consider the following parameters dependent Lyapunov-Krasovskii functional:

$$
\begin{aligned}
& V\left(z_{t}, \alpha\right)=z_{t}^{T}(0) P(\alpha) z_{t}(0)+\int_{-h(t)}^{0} z_{t}^{T}(\theta) Q(\alpha) z_{t}(\theta) d \theta+\int_{-h_{m}}^{0} z_{t}^{T}(\theta) Q_{m}(\alpha) z_{t}(\theta) d \theta \\
& +\int_{-h_{m}}^{0} \int_{\theta}^{0} \dot{z}_{t}^{T}(s) R(\alpha) \dot{z}_{t}(s) d s d \theta+\int_{-h_{m}}^{0} \int_{s}^{0} \int_{u}^{0} \ddot{x}_{t}^{T}(\theta) W(\alpha) \ddot{x}_{t}(\theta) d \theta d u d s
\end{aligned}
$$

where $P(\alpha), Q(\alpha)$ and $R(\alpha) \in \mathrm{R}^{2 \mathrm{n} \times 2 \mathrm{n}}$ and $W(\alpha) \in \mathrm{R}^{\mathrm{n} \times \mathrm{n}}$ are positive definite parameters dependent Lyapunov matrices.

First, as previously, let define the two matrices $E_{1}=\left[\begin{array}{ll}1_{n} & 0_{n}\end{array}\right]$ and $E_{2}=$ $\left[\begin{array}{ll}0_{n} & 1_{n}\end{array}\right]$. Considering the Lyapunov-Krasovskii functional (46) and introducing once again signals (33) leads to the inequality

$$
\begin{aligned}
\dot{V} \leq & 2 \dot{z}^{T}(t) P(\alpha) z(t)+z^{T}(t) Q(\alpha) z(t)-(1-\dot{h}(t)) z^{T}(t-h(t)) Q(\alpha) z(t-h(t)) \\
& +h_{m} \dot{z}^{T}(t) R(\alpha) \dot{z}(t)-h(t) \delta_{1}^{T}(t) R(\alpha) \delta_{1}(t)+\frac{h_{m}^{2}}{2} \dot{z}^{T} E_{2}^{T} W(\alpha) E_{2} \dot{z}-2 \delta_{2}^{T}(t) E_{1}^{T} W(\alpha) E_{1} \delta_{2}(t) \\
& -z^{T}\left(t-h_{m}\right) Q_{m}(\alpha) z\left(t-h_{m}\right)-\left(z(t-h(t))-z\left(t-h_{m}\right)\right)^{T} \frac{R(\alpha)}{h_{m}}\left(z(t-h(t))-z\left(t-h_{m}\right)\right) .
\end{aligned}
$$

Defining the extended vector:

$$
\xi(t)=\left[\begin{array}{c}
z(t) \\
\dot{z}(t) \\
z(t-h(t)) \\
z\left(t-h_{m}\right) \\
w(t) \\
\delta_{1}(t) \\
\delta_{2}(t)
\end{array}\right],
$$

expression (47) can be rewritten as

$$
\dot{V} \leq \xi^{T}(t) \Gamma(\alpha) \xi(t)
$$

with

$\Gamma(\alpha)=\operatorname{diag}\left(\left[\begin{array}{cc}Q(\alpha) & P(\alpha) \\ P(\alpha) & T(\alpha)\end{array}\right],\left[\begin{array}{cc}-(1-d) \mathbf{Q}^{[i]}-\frac{1}{h_{m}} R^{[i]} & \frac{1}{h_{m}} R^{[i]} \\ \frac{1}{h_{m}} R^{[i]} & -Q_{m}^{[i]}-\frac{1}{h_{m}} R^{[i]}\end{array}\right], 0_{2 \mathrm{n}},-\mathrm{h}_{\mathrm{m}} \mathrm{R}(\alpha),-2 \mathrm{E}_{1}^{\top} \mathrm{W}(\alpha) \mathrm{E}_{1}\right)$

with $T(\alpha)=h_{m} R(\alpha)+\frac{h_{m}^{2}}{2} E_{2}^{T} W(\alpha) E_{2}$. Note that from inequality (47) to inequality (49), variables $h(t)$ and $\dot{h}(t)$ have been bounded by $h_{m}$ and $d$ respectively. This operation introduces some conservatism but is necessary to avoid bilinear terms between parameters. Thus, the condition of a decreasing Lyapunov functional along the trajectories of system (43) can be described by

$$
\xi^{T}(t) \Gamma(\alpha) \xi(t)<0 \quad \text { s.t. } \mathrm{S}(\alpha, \mathrm{h}, \dot{\mathrm{h}}) \xi(\mathrm{t})=0 .
$$


where matrix $S \in \mathbb{R}^{9 n \times 14 n}$ specifies relationships between the components of $\xi(t)$ :

$$
S=\left[\begin{array}{ccccccc}
\bar{A}(\alpha) & -E & \bar{A}_{d 1}(\alpha) & 0 & \bar{A}_{d 2}(\alpha) & 0 & 0 \\
0 & 0 & \bar{h}(t) 1 & 0 & 1 & 0 & 0 \\
1 & 0 & -1 & 0 & 0 & -h(t) 1 & 0 \\
0 & 1 & 0 & 0 & 0 & -1 & -1
\end{array}\right]
$$

Invoking Finsler's lemma, if there exists $X \in \mathrm{R}^{14 \mathrm{n} \times 9 \mathrm{n}}$ such that

$$
\Gamma(\alpha)+X S(\alpha, h, \dot{h})+S^{T}(\alpha, h, \dot{h}) X^{T}<0,
$$

hold then the former condition is satisfied. Note that the matrix $S$ can be written as $S(\alpha)=\sum_{i=1}^{\eta} \alpha_{i} S^{[i]}$ where $S^{[i]}$ are the vertices of $S$ :

$$
S^{[i]}=\left[\begin{array}{ccccccc}
\bar{A}^{[i]} & -E & \bar{A}_{d 1}^{[i]} & 0 & \bar{A}_{d 2}^{[i]} & 0 & 0 \\
0 & 0 & \dot{h}(t) 1 & 0 & 1 & 0 & 0 \\
1 & 0 & -1 & 0 & 0 & -h(t) 1 & 0 \\
0 & 1 & 0 & 0 & 0 & -1 & -1
\end{array}\right] .
$$

with $\bar{A}^{[i]}, \bar{A}_{d 1}^{[i]}$ and $\bar{A}_{d 2}^{[i]}$ are defined in (45). Consequently, the inequality (51) becomes

$$
\sum_{i=1}^{\eta} \alpha_{i}\left(\Gamma^{[i]}+X S^{[i]}(h, \dot{h})+S^{[i]^{T}}(h, \dot{h}) X^{T}\right)<0,
$$

for all $\alpha_{i}>O$ such that $\sum_{i=1}^{\eta} \alpha_{i}=1$ with $\Gamma^{[i]}$ defined as (45). In order to ensure inequality (52), it is equivalent to test

$$
\Gamma^{[i]}+X S^{[i]}(h, \dot{h})+S^{[i]^{T}}(h, \dot{h}) X^{T}<0, \quad \forall \mathrm{i}=\{1, \ldots, \eta\} .
$$

By embedding $h(t)$ and $\dot{h}(t)$ into a polytopic set and using quadratic stability framework, a sufficient condition to assess the definite negativity of (53) is

$$
\Gamma^{[i]}+X S^{[i][j][k]}+S^{[i][j][k]^{T}} X^{T}<0
$$

for all $i=\{1, \ldots, \eta\}, j=\{1,2\}$ and $k=\{1,2\}$, with $S^{[i][j][k]}$ is defined as (45). If the latter condition is satisfied then the system (43) is asymptotically robustly stable. As previously, since the whole state $z$ converges asymptotically to zero, its first component $x$ converges as well for all $\alpha \in \Xi$ (41).

Following the same methodology than previously, based on Theorem 2, again a robust stability criterion can be derived:

Theorem 5 Given scalars $h_{m}>0, d \geq 0$, the linear uncertain system (40) is asymptotically robustly stable for any time-varying delay $h(t)$ satisfying (2), (3) and $\alpha \in \Xi$ (41) if there exists definite positive matrices $P^{[i]}, Q_{1}^{[i]}, Q_{2}^{[i]}$, $R^{[i]} \in \mathrm{R}^{2 \mathrm{n} \times 2 \mathrm{n}}$ and a matrix $X \in \mathrm{R}^{8 \mathrm{n} \times 3 \mathrm{n}}$ such that the following LMI holds for $i=\{1, \ldots, \eta\}, j=\{1,2\}$ :

$$
\boldsymbol{\Gamma}^{[i][j]}+\mathbf{X} S^{[i][j]}+S^{[i][j]^{T}} \mathbf{X}^{T}<0
$$


where

$$
\begin{aligned}
& S^{[i][j]}=\left[\begin{array}{llll}
-E & A^{[i]} & A_{d}^{[i][j]} & 0
\end{array}\right] \\
& \Gamma^{[i][j]}=\left[\begin{array}{cccc}
h_{m} \mathbf{R}^{[i]} & \mathbf{P}^{[i]} & 0 & 0 \\
\mathbf{P}^{[i]} & T^{[i]} & \frac{1}{h_{m}} \mathbf{R}^{[i]} & 0 \\
0 & \frac{1}{h_{m}} \mathbf{R}^{[i]} & U^{[i][j]} & \frac{1}{h_{m}} \mathbf{R}^{[i]} \\
0 & 0 & \frac{1}{h_{m}} \mathbf{R}^{[i]} & V^{[i]}
\end{array}\right] \begin{aligned}
T & =\mathbf{Q}_{\mathbf{1}}{ }^{[i]}+\mathbf{Q}_{\mathbf{2}}{ }^{[i]}-\frac{1}{h_{m}} \mathbf{R}^{[i]} \\
\text { with } & =-\left(1-\dot{h}^{[j]}\right) \mathbf{Q}_{\mathbf{1}}{ }^{[i]}-\frac{2}{h_{m}} \mathbf{R}^{[i]}, \\
V & =-\frac{1}{h_{m}} \mathbf{R}^{[i]}-\mathbf{Q}_{2}{ }^{[i]}
\end{aligned}, \\
& \bar{A}^{[i]}=\left[\begin{array}{cc}
A^{[i]} & 0 \\
0 & A^{[i]} \\
0 & 1
\end{array}\right], \quad \bar{A}_{d}^{[i][j]}=\left[\begin{array}{cc}
A_{d}^{[i]} & 0 \\
0 & \left(1-\dot{h}^{[j]}\right) A_{d}^{[i]} \\
0 & 0
\end{array}\right], \quad\left\{\begin{array}{l}
\dot{h}^{[1]}=-d \\
\dot{h}^{[2]}=d
\end{array}\right.
\end{aligned}
$$

with $A^{[i]}$ and $A_{d}^{[i]}$ for $i=\{1, \ldots, \eta\}$ are the vertices of the matrices $A(\alpha)$ and $A_{d}(\alpha)$ according to the polytopic uncertainty.

\section{Proof 5 Omitted}

\section{$5 \quad$ Numerical examples}

\subsection{First example}

Consider the following system,

$$
\dot{x}(t)=\left[\begin{array}{cc}
-2 & 0 \\
0 & -0.9
\end{array}\right] x(t)+\left[\begin{array}{cc}
-1 & 0 \\
-1 & -1
\end{array}\right] x(t-h(t)) .
$$

For this academic example, we want to assess the maximal allowable delay for different values of $d$. To demonstrate the effectiveness of our criterion, results are compared against those obtained in [5], [6], [19], [9], [10] and [11]. All these papers, except the last one, use the Lyapunov theory in order to derive some stability analysis criteria for time delay systems. In [11], the stability problem is solved by a classical robust control approach: the IQC framework. The results are shown in Table 1.

The numerical experiments show that Theorem 1 gives similar results to [9]. That is logical since the same Lyapunov functional is used. Results for $d \geq 1$ and $\forall d$ are computed with Theorem 1 and choosing $Q_{1}=0$ in (6). [6] gives a rate-independent criterion which is interesting when $d$ is unknown or for fast-varying delays. However, this approach is very conservative when a bound on $\dot{h}(t)$ is known.

Then, considering the augmented system (15) composed by the original system (1) and its derivative, Theorem 2 improves the maximal allowable delays for $0 \leq d \leq 1$ (since the proposed approach is rate-dependent, it does not provide conditions independent of $d$ ). Indeed, using the same Lyapunov-Krasovskii functional, conservatism is reduced thanks to the derivative of (1). As expected, this operation provides more information on the system and thus improves the stability analysis criterion.

Furthermore, Theorem 3 which consider an additional term (29) improves again the upperbound $(\forall d \in[0,1])$. This result suggests that the new proposed Lyapunov-Krasovskii functional (29) is suitable for time varying delay system 
Table 1: The maximal allowable delays $h_{m}$ for system (56)

\begin{tabular}{|c||c|c|c|c|c|c|c|}
\hline $\mathrm{d}$ & 0 & 0.1 & 0.2 & 0.5 & 0.8 & 1 & $\forall d$ \\
\hline \hline$[5]$ & 4.472 & 3.604 & 3.033 & 2.008 & 1.364 & 0.999 & 0.999 \\
\hline$[6]$ & 1.632 & 1.632 & 1.632 & 1.632 & 1.632 & 1.632 & 1.632 \\
\hline$[19]$ & 4.472 & 3.604 & 3.033 & 2.008 & 1.364 & - & - \\
\hline$[11]$ & 4.472 & 3.604 & 3.033 & 2.008 & 1.364 & 0.999 & - \\
\hline$[9]$ & 4.472 & 3.605 & 3.039 & 2.043 & 1.492 & 1.345 & 1.345 \\
\hline$[10]$ & 4.472 & 3.605 & 3.039 & 2.043 & 1.492 & 1.345 & 1.345 \\
\hline Theorem 1 & 4.472 & 3.605 & 3.039 & 2.043 & 1.590 & 1.345 & 1.345 \\
\hline Theorem 2 & 4.472 & 3.670 & 3.209 & 2.514 & 2.181 & 2.034 & - \\
\hline Theorem 3 & 5,120 & 4,081 & 3,448 & 2,528 & 2,152 & 1,991 & - \\
\hline
\end{tabular}

stability analysis, reducing conservatism. Lines means that no results have been found.

Remark 5 Theorem 2 and 3 provide both good results. However, it can be observed that in example (56) for $|\dot{h}| \geq 0.8$, Theorem 2 provides slightly better results than Theorem 3. This difference could be explained by the use in the LKF of Theorem 2 of the term $\int_{t-h_{m}}^{t} z^{T} Q_{2} z$ and applying the separation of the integral in the third term as (9). Consequently, it seems that the additional term (29) reduce conservatism when slow time-varying delays are considered.

\subsection{Second example}

Consider the following system,

$$
\dot{x}(t)=\left[\begin{array}{cc}
0 & 1 \\
-1 & -2
\end{array}\right] x(t)+\left[\begin{array}{cc}
0 & 0 \\
-1 & 1
\end{array}\right] x(t-h(t)) .
$$

The delay dependent stability analysis of system (57) has been studied and results are shown in table (2). System (57) is IOD stable (independent of delay) when the delay is constant. Once again, it is observed that Theorem 2 and Theorem 3 improves the maximal bound on the delay which preserves the stability of $(57)$.

\subsection{Third example}

Consider the following uncertain system,

$$
\dot{x}(t)=\left[\begin{array}{cc}
0 & -0.12+12 \rho \\
1 & -0.465-\rho
\end{array}\right] x(t)+\left[\begin{array}{cc}
-0.1 & -0.35 \\
0 & 0.3
\end{array}\right] x(t-h(t)) .
$$

where $\rho$ is an uncertain parameter such that $|\rho| \leq 0.035$. Applying Theorem 4 and 5 to assess the delay dependent stability of system (58), corresponding maximal delays $h_{m}$ for different values of $d$ are given in Table 3 
Table 2: The maximal allowable delays $h_{m}$ for system $(57)$

\begin{tabular}{|c||c|c|c|c|c|}
\hline $\mathrm{d}$ & 0 & 0.1 & 0.2 & 0.5 & 0.8 \\
\hline \hline$[5]$ & $\infty$ & 5.459 & 3.25 & 0.999 & 0.560 \\
\hline$[6]$ & 1.082 & 1.082 & 1.082 & 1.082 & 1.082 \\
\hline$[19]$ & $\infty$ & 5.459 & 3.255 & 0.999 & 0.560 \\
\hline$[9]$ & $\infty$ & 5.461 & 3.264 & 1.082 & 0.774 \\
\hline Theorem 2 & $\infty$ & 5.823 & 3.824 & 2.0083 & 1.356 \\
\hline Theorem 3 & $\infty$ & 6.320 & 3.949 & 1.995 & 1.332 \\
\hline
\end{tabular}

Table 3: The maximal allowable delays $h_{m}$ for system (58)

\begin{tabular}{|c||c|c|c|c|c|}
\hline $\mathrm{d}$ & 0 & 0.1 & 0.2 & 0.5 & 0.8 \\
\hline \hline Theorem 4 & 1.475 & 1.444 & 1.426 & 1.395 & 1.380 \\
\hline Theorem 5 & 0.875 & 0.800 & 0.740 & 0.617 & 0.544 \\
\hline
\end{tabular}

\section{CONCLUSION}

In this paper, the problem of the delay dependent stability analysis of a time varying delay system has been studied using Lyapunov-Krasovskii functionals. The first criterion is based on an existing Lyapunov-Krasovskii functional [9] (see Theorem 1). Based on this first result, and using an augmented state, new types of Lyapunov-Krasovskii functional are introduced which emphasizes the relation between $\dot{h}$ and signals $\dot{x}$ and $\ddot{x}$. The resulting criteria are then expressed in terms of a convex optimization problem with LMI constraints, allowing for the use of efficient solvers. Moreover, it is shown that the derived stability condition reduces conservatism in the sense that it can not provide worse results than traditional ones in the literature. Finally, numerical examples show that these methods improved the maximal allowable delay compared to the results of the literature.

\section{References}

[1] Y. Ariba and F. Gouaisbaut. Delay-dependent stability analysis of linear systems with time-varying delay. In IEEE Conference on Decision and Control, pages 2053-2058, December 2007.

[2] P.-A. Bliman. Lyapunov equation for the stability of linear delay systems of retarded and neutral type. IEEE Trans. on Automat. Control, 47:327-335, February 2002.

[3] S. Boyd, L. El Ghaoui, E. Feron, and V. Balakrishnan. Linear Matrix Inequalities in System and Control Theory. SIAM, Philadelphia, USA, 1994. in Studies in Applied Mathematics, vol.15.

[4] Y. Ebihara, D. Peaucelle, D. Arzelier, and T. Hagiwara. Robust performance analysis of linear time-invariant uncertain systems by taking higherorder time-derivatives of the states. In $44^{\text {th }}$ IEEE Conference on Decision 
and Control and the European Control Conference, Seville, Spain, December 2005 .

[5] E. Fridman and U Shaked. An improved stabilization method for linear time-delay systems. IEEE Trans. on Automat. Control, 47:1931-1937, November 2002.

[6] E. Fridman and U Shaked. Input-output approach to stability and $l_{2}$-gain analysis of systems with time-varying delays. Systems $\&$ Control Letters, 55:1041-1053, September 2006.

[7] F. Gouaisbaut and D. Peaucelle. Delay-dependent robust stability of time delay systems. In $5^{\text {th }}$ IFAC Symposium on Robust Control Design (ROCOND'06), Toulouse, France, July 2006.

[8] K. Gu, V. L. Kharitonov, and J. Chen. Stability of Time-Delay Systems. Birkhäuser Boston, 2003. Control engineering.

[9] Y. He, Q. G. Wang, C. Lin, and M. Wu. Delay-range-dependent stability for systems with time-varying delay. Automatica, 43:371-376, 2007.

[10] Y. He, Q. G. Wang, L. Xie, and C. Lin. Further improvement of freeweighting matrices technique for systems with time-varying delay. IEEE Trans. on Automat. Control, 52:293-299, February 2007.

[11] C. Y. Kao and A. Rantzer. Robust stability analysis of linear systems with time-varying delays. In $16^{\text {th }}$ IFAC World Congress, Prague, Czech Republic, 2005.

[12] V. B. Kolmanovskii and J. P. Richard. Stability of some linear systems with delays. IEEE Trans. on Automat. Control, 44:984-989, May 1999.

[13] S.I. Niculescu. Delay Effects on Stability. A Robust Control Approach, volume 269 of Lecture Notes in Control and Information Sciences. SpringerVerlag, Heildelberg, 2001.

[14] N. Olgac and R. Sipahi. An exact method for the stability analysis of time-delayed linear time-invariant (lti) systems. IEEE Trans. on Automat. Control, 47(5):793-797, 2002.

[15] D. Peaucelle and F. Gouaisbaut. Discussion on parameter-dependent lyapunov functions approach to stability analysis and design for uncertain systems with time-varying delay. European J. of Control, 11(1):69-70, 2005.

[16] J.-P. Richard. Time-delay systems: an overview of some recent advances and open problems. Automatica, 39:1667-1694, October 2003.

[17] Rifat Sipahi and Nejat Olgac. Stability robustness of retarded lti systems with single delay and exhaustive determination of their imaginary spectra. SIAM J. Control Optim., 45(5):1680-1696, 2006.

[18] R. Skelton, T. Iwazaki, and K. Grigoriadis. A unified algebric approach to linear control design. Taylor and Francis series in systems and control, 1998.

[19] M. Wu, Y. He, J. H. She, and G. P. Liu. Delay-dependent criteria for robust stability of time-varying delay systems. Automatica, 40:1435-1439, 2004. 